Review

\title{
New Metabolic and Genetic Biomarkers for Diagnosis of Ulcerative Colitis
}

\author{
Elena-Emilia Tudoroiu1, Mihaela-Andreea Costache ${ }^{1}$ and Maria-Andreea Stancu ${ }^{1, *}$ and Anca Lucia Pop ${ }^{1}$ \\ 1 Department of Clinical Laboratory, Food Safety, "Carol Davila" University of Medicine and Pharmacy, 6 \\ Traian Vuia Street, Bucharest 020945, Romania, elena.tudoroniu@rez.umfcd.ro, \\ mihaela.costache@rez.umfcd.ro, maria.stancu@rez.umfcd.ro, anca.pop@umfcd.ro \\ * Correspondence: maria.stancu@rez.umfcd.ro (M.S.); anca.pop@umfcd.ro (A.L.P.) Tel.: +40745009910
}

\begin{abstract}
Ulcerative colitis (UC) is one of the two disorders known as inflammatory bowel diseases (IBD) along with Crohn's disease (CD), with complex pathogenesis, requiring costly invasive investigations. Objective: to examine the most recent biomarkers proposed for UC diagnosis; to establish the strategy used to make the differential diagnosis between UC and CD relying on these biomarkers, also adding the benefit of finding new non-invasive tools in managing this condition. The search was performed in a single database (Web of Science) using the specific keywords ",ulcerative colitis", „biomarkers" and "diagnosis” for the last five years. Study eligibility criteria: clinical trials on adults and pediatric patients with ulcerative colitis compared with Crohn's disease. Results: We selected 57 studies, randomized controlled trials (RCTs) and clinical case series (CCS), summarizing the latest most specific biomarkers in diagnosis of UC. Limitations: we considered RCTs and CCS from one database, limited to the search topics. Our findings indicate a important number of potential biomarkers with diagnostic value, which bring the advantage of a non-invasive method to approach this challenging disorder.
\end{abstract}

Keywords: ulcerative colitis; biomarkers; diagnosis; inflammatory bowel disease; Crohn's disease

\section{Introduction}

Inflammatory bowel diseases (IBD) consist of two major clinical phenotypes: ulcerative colitis (UC) and Crohn's disease (CD) [1]. Europe has the highest incidence of IBD, with 2.5-3 million people affected [2], while in North America there are 1.5 million people with IBD [3].

UC is an inflammatory bowel disease, a chronic condition, associated with modified gut microbiota, with unexplained causes [4,5]. It presents alternative periods of remission and relapse [6,7]. In this condition, the most affected is the mucosa of colon and rectum [8]. The most frequent symptom is bloody diarrhea, but this pathology is also manifested by other disorders, such as vomiting, abdominal pain, weight loss and fever, which contribute to an improper quality of life [9]. The first symptoms of nearly $20-30 \%$ of individuals with IBD appear before the age of 18 years [10]. During last years, the global prevalence has enhanced promptly, with a higher incidence at women. UC is the result of a complex of external, genetic, immunological and inflammatory factors [11], but it has been found that the major inflammatory compound in active UC are the neutrophils from the gut mucosa [12]. Many patients with UC have also other autoimmune diseases, e.g. rheumatoid arthritis, rheumatoid spondylitis, nodular erythema or sclerosis cholangitis. Thus, an early diagnosis of UC has a huge impact on patient's life, efficient treatments can be administrated to mitigate the patient suffering, to improve the quality of life [13], and to prevent the major complication of UC, colorectal cancer [14]. 
The prevalence of UC in children is between 0.5 and 4.3 per 100,000 individuals [15] and the symptoms are more aggressive than adults [16].

Both diseases are characterized by similar symptoms, but different treatment procedures; therefore, an accurate diagnosis is mandatory. UC is characterized by continuous inflammatory changes within the mucous membrane and affects only the large intestine [17]. The incidence of IBD is increasing in newly industrialized nations, being associated with consumption of processed food and low fibers intake, but the clear etiology of IBD still remains unknown. The first incriminated cause is the role of heredity pathogenesis; secondly, the abnormal interactions between the host immune system and gut flora and, as well as, dysregulation of cellular responses, such as autophagy and endoplasmic reticulum stress, resulting in intestinal inflammation. Also a possible reason is represented by the influence of other genetic, especially epigenetic changes, including microRNA-mediated regulation of gene expression [18].

The purpose of this review was to identify the newest biomarkers for diagnosis of $\mathrm{UC}$, and also for differentiation between $\mathrm{UC}$ and $\mathrm{CD}$ in the patients with IBD, the major aim being the premature diagnosis of these diseases to prevent subsequent complications and to improve the quality of the patient life.

\section{Materials and Methods}

In the present paper, we performed a systematic qualitative review according to the Preferred Reporting Items for Systematic Review and Meta-Analysis (PRISMA) guidelines searching original published papers on new potential biomarkers for diagnosis of ulcerative colitis; other criteria included: published in a scholarly peerreviewed journal; written in English and from the last five years.

The review methods of the search were established in advance. The search was achieved in one major database, Web of Science Core Collection. The report had no significant deviations from the initial study plan.

Data sources: we searched the Web of Science database for ulcerative colitis, biomarkers, diagnosis and other related keywords. Search: we did a search in one database (filters applied: clinical trial, randomized controlled trial in the last five years) with the keywords: "ulcerative colitis" AND "biomarkers" AND "diagnosis"; we restricted searching for articles written in English; the last updated search was done on 12 July 2021.

Study selection: inclusion criteria were: patients with a diagnosis of IBD or UC or with an inflammatory status of gut microbiota in randomized clinical trials (RCTs) and clinical case series (CCS) published in the last five years.

Data extraction: the following data were selected: author(s), year of publication, country and aim of the study, study design, and main results. We selected RCTs and CCS as presented below for all the data searched and retrieved from the database sources. Firstly, the authors screened articles by title and abstract, and then by full text. Duplicates and articles not fulfilling the search criteria were excluded.

Data analysis was performed by three authors (E.E.T., M.A.C., and M.A.S.). 163 studies with biomarkers in ulcerative colitis or with biomarkers for diagnosis of IBD or with biomarkers for differentiation between UC and CD were identified and screened for eligibility by the three examinators. We completed the data collection in July 2021. The quality of the studies selected for review was evaluated.

Fifty-seven papers were included in the present study, centered on the three main topics included in the search. 


\section{Results}

\subsection{Study Selection}

We searched for: TITLE: (Ulcerative colitis diagnosis) Refined by TITLE: (biomarkers) AND DOCUMENT TYPES:(ARTICLE). Timespan: 2017-2021 (last five years). Results: 163 articles.

From the databases, we selected 57 studies for full-text reading, after initial retrieving of 163 articles, screened by title and abstract or in extenso to match the search criteria, and exclusion of duplicates; the screened research was centered on twelve main topics in the UC biomarkers published research: (a) fecal analysis biomarkers, (b) different stages of disease biomarkers, and (c) organs biomarkers in UC, (d) $\mathrm{CRP} /$ albumin ratio, (e) microRNAs, (f) galectins, (g) gut microbiota biomarkers in UC, (h) autoantibodies (i) volatile organisc compounds, (j) protein linked carbohydrates, (h) serum fetuin, (i) transferrin and (j) other potential biomarkers . The selection process is presented in the PRISMA flow diagram (Figure 1).

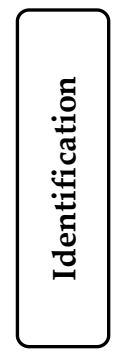

We did a Web of Science ${ }^{\circledR}$ data search for RCT/CT in the last 5 years using the following keywords: "ulcerative colitis" and "biomarkers" and "diagnosis"

Records identified from Web of Science $(n=163)$

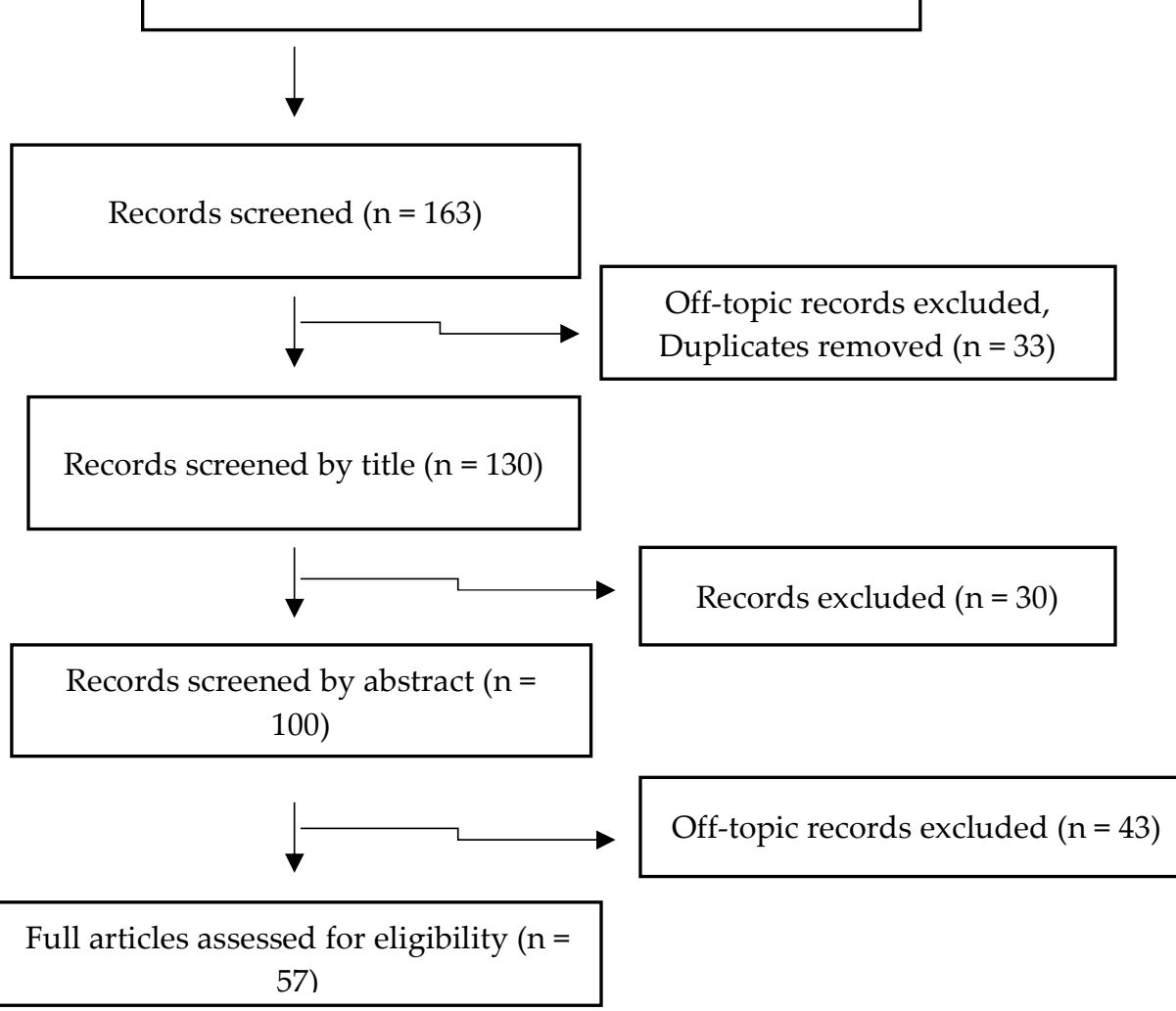

Figure 1. Preferred Reporting Items for Systematic Review and Meta-Analysis (PRISMA) diagram describing a systematic search and study selection process. 


\subsection{Biomarkers for diagnosis of UC}

One of the biggest challenges is to discover new non-invasive biomarkers with a higher sensitivity and specificity to detect as early as possible the inflammation of the gut mucosa to prevent the appearance of clinical symptoms and, thus, to prevent the progressive impairment of gastrointestinal mucosa [19].

Because of its unexplained etiology, UC evaluation requires a variety of medical tests and procedures, including laboratory analysis, histopathological investigation, imaging examination and genetic tests. Regarding laboratory analysis, inflammatory biomarkers are essential for the assessment of acute phase of UC, because they are commonly used, they are cheap, they have a non-invasive character, and they are nontraumatic for the patient. In this category, we found CRP, ESR, platelets, fibrinogen, ferritin, and albumin, indicators that are investigated in the patient plasma. The major drawbacks of these markers are the low sensitivity and specificity [20].

If we talk about fecal markers, the main parameter used to evaluate the UC activity, different stages of its evolution, the mucosal healing, the response to treatment or the necessity of a surgery is fecal calprotectin (FC) [21]. It is able to differentiate between IBD and irritable bowel syndrome, but not for the differentiation of UC from CD. There are more other promising fecal parameters that are intensely studied, such as neutrophil gelatinase associated lipocalin, cathelicidins, beta-glucuronidase and osteoprotegerin $[22,23]$.

\subsubsection{Fecal analysis}

A prospective cohort study led by Galipeau et al. showed that a heightened level of fecal proteolytic activity could be an early non-invasive biomarker for gut inflammation, which could be monitored in the predisposed population to ulcerative colitis (UC), before the clinical diagnosis for this pathology. During this study, there were applied several types of analysis, such as shotgun metagenomic and 16S rRNA gene sequencing, gnotobiotic colonization, and in vitro functional examination to describe the microbial composition in fecal samples, collected from pre-UC, post-UC, and healthy individuals. The study illustrated different changes in the composition of gut microbiota for pre-UC subjects, which showed higher levels for pathogens (Actinobacillus) and lower levels for favorable bacteria (Adlercreutzia, with anti-inflammatory properties and Bifidobacillus). The pre-UC population presents a large production of proteases and peptidases by intestinal bacteria (Bacteroides vulgates and Parabacteroides) and an augmented metabolism of proteins. Moreover, the study illustrated an increased level of elastase activity in Bacteroides, isolated from fecal samples. Consequently, the study opened different pathways for early investigation of predisposed people to UC and also for possible treatments by reduction of high proteolytic activity [24].

Calprotectin, a 36-kDa glycoprotein and a Ca- and Zn-binding protein, from lipocalin family, is found in the cytoplasm of inflammatory cells such as neutrophils, macrophages, and activated monocytes. The concentration of calprotectin in stool is proportional to the number of neutrophils in the intestinal lumen, making this marker more specific and sensitive for detecting inflammation in the digestive tract than other serological markers. Also, calprotectin offers the advantage of detecting inflammation at an early stage, before other non-specific markers such as the erythrocyte sedimentation rate (ESR), C-reactive protein (CRP), and orosomucoid can be detected [25].

A new perspective for this marker is the prediction of endoscopic activity in inflammatory bowel disease. With this notion comes the "mucosal healing", an important therapeutic goal in Crohn's Disease and Ulcerative Colitis (UC). This can be defined as the "complete absence of all inflammatory and ulcerative lesions in all segments of gut" at endoscopy. As repeating colonoscopy is not feasible owing to the costs and potential adverse effects and clinical signs frequently do not correlate with 
endoscopic indices or real inflammatory activity, finding a marker to predict the presence of endoscopic lesions is a key in inflammatory bowel disease diagnosis [26].

Jusue et al. showed the value of calprotectin as a predictor of endoscopic activity in IBD and that it is more accurate in ulcerative colitis than in Crohn's disease. The greater accuracy of the marker in UC was based on the fact that calprotectin was more sensitive for detecting colonic lesions than ileal lesions. Also higher levels of fecal calprotectin in patients with no evidence of endoscopic activity suggested that endoscopy may underestimate the disease activity and the need of another route of monitoring. The study also reported that the fecal calprotectin concentration in UC was closely associated with the severity of endoscopic lesions [26].

Fecal calprotectin (f-Cp) has high sensitivity and specificity for discriminating between IBD and functional bowel disorders in adults and children. Moreover, $\mathrm{f}-\mathrm{Cp}$ is more sensitive and specific for IBD than commonly used inflammatory markers, and its levels link with the degree of neutrophilic infiltration. F-Cp may also be a helpful tool for monitoring disease progression and response to therapy and predicting relapse and risk of bowel resection [27].

Whitehead et al. conducted a study that has as main aim to evaluate whether between-assay variability of different fecal calprotectin (f-Cp) assays influences diagnostic accuracy in a cohort of patients and brought up the fact that this inter-assay variability can be due to the absence of standardization. They report a prospective clinical evaluation in which four commercially available $\mathrm{f}-\mathrm{Cp}$ assays were compared to assess whether inter-assay variability influences clinical decision-making, and the diagnostic performance of the different $\mathrm{f}-\mathrm{Cp}$ assays was similar despite inter-assay variability in same sample absolute $\mathrm{f}-\mathrm{Cp}$ values. The conclusion of the study was that there is a necessity for assay standardization, but in its absence they suggest that assayspecific cut-off values may optimize their diagnostic performance and second that clinicians be made aware that $\mathrm{f}-\mathrm{Cp}$ results from different assays are not interchangeable [27].

Another study which evaluated the performance of combinations of fecal and blood markers on the detection of endoscopically active disease showed that combining FCT and fecal immunochemical test improved the specificity to $95 \%$, but the sensitivity decreased to $65.4 \%$ [28].

\subsubsection{Markers for different stages of UC}

During last years, many researchers have been working to discover other biomarkers for diagnosis of UC, using many analysis techniques, from blood and fecal analysis (C-reactive protein, erythrocyte sedimentation rate and fecal calprotectin - the most common analysis for UC diagnosis), to genomic and metabolomic analysis [29].

Another method of examination is peptidomics, which defines the qualitative and quantitative analysis of peptides with low molecular weight (LMW) in different biological samples. For example, Miao et al. managed such a study with 78 people, including 26 with active UC, 26 with remitting UC and 26 healthy people. Researchers applied a nano-liquid chromatography coupled with hybrid linear ion trap Orbitrap (Nano LC-LTQ-Orbitrap) mass spectrometry to evaluate the serum peptidome of all 78 subjects and there were identified 40 peptides, but only 6 biomarker peptides presented adequate features for curve analysis. These 6 peptides could be important biomarkers to differentiate the subjects with active UC from subjects with remitting UC and healthy subjects; thus, serum peptidomics represents a new method to discover new markers for UC diagnosis and for the differentiation of different phases of UC [29]. 


\subsubsection{Biomarkers from different organs}

In a study led by Hong et al., which demonstrated the effect of Compound Sophorae Decoction in UC, there were identified 36 potential biomarkers, using mass spectrometry. These metabolites were found in serum, urine, colon, kidney and liver, with various levels in UC, higher or lower [13], all this information being reviewed in Table 1.

Table 1. Potential biomarkers and their level in ulcerative colitis [13].

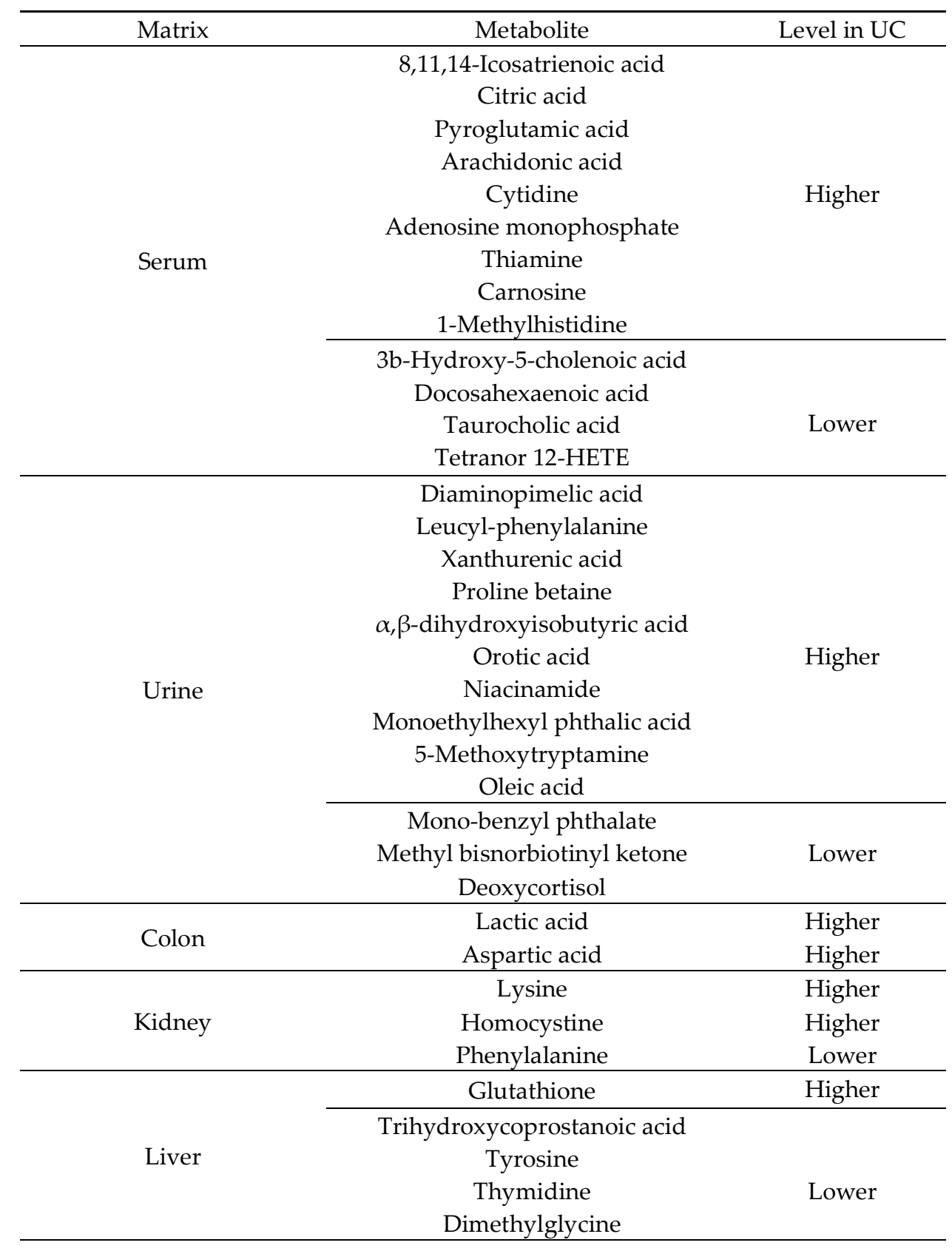

\subsubsection{CRP/Albumin ratio (CAR)}

CAR or CRP to albumin ratio is an anti-inflammatory biomarker, which has been studied extensively during last years and studies showed that this is a useful indicator to 
diagnose many types of cancer, acute pancreatitis or sepsis. An enhanced activity of inflammation is associated with a high level of CAR.

Although colonoscopy is the main and the conclusive medical procedure for UC diagnosis, it is important to discover and to identify other parameters that can be used more easily than a colonoscopy, a complex procedure for patient and doctor. Regarding biomarkers from blood and feces, the most used for UC investigation are CRP and fecal calprotectin [30].

CRP is a protein that characterizes the acute stage of an anti-inflammatory disease and it is the response to pro-inflammatory cytokines, such as interleukin-1 (IL-1), interleukin-6 (IL-6) and TNF alpha (TNF- $\alpha$ ), but it is a nonspecific parameter, especially if UC is in a moderate stage of symptoms. Nevertheless, CRP is a superior marker to procalcitonin, erythrocyte sedimentation rate and albumin for UC diagnosis. Albumin is the opposite indicator of CRP and it is also related to the nutritional status of the patient. Therefore, an anti-inflammatory response is the outcome of an increased level of CRP and a decreased level of albumin. The combination between CRP and albumin is called CAR and it indicates the mixture of systemic swelling and nutritional situation. A recent study realized on patients with active UC illustrated that CAR had a higher specificity and AUC value comparative to CRP and albumin. It is a simple, low-cost and quickly determined indicator, so it can be a useful biomarker due to this characteristics in the diagnosis of severe UC [30].

\subsubsection{MicroRNAs}

Circulating microRNAa (miRNAs) is a hopeful non-invasive biomarker for IBD diagnosis. It is a small noncoding RNAs, consisting of 18-25 nucleotides with an essential role in different biological processes and it is found in serum, saliva, urine and plasma. Since 2008, miRNAs is extremely studied asa a potential biomarker for many conditions, including IBD. From all miRNAs genes, a specific role in down-regulation of patients with UC have miR-143 and miR-145, identified in the colon biopsies of this subjects and miR-7, found in the CD subjects mucosa [31].

MicroRNAs are important post-transcriptional players that regulate the expression of genes during the inflammation and thus could be appropriate biomarkers for differentiation between UC and CD.

Guz et al. confirmed in a study that the lower expression of E-cadherin (CDH1) in patients with $\mathrm{CD}$, but not in patients with $\mathrm{UC}$, could evidence the involvement of this protein in $\mathrm{CD}$ pathogenesis. Also downregulated $\mathrm{CDH} 1$ was negatively correlated with miR-155-5p expression in both the inflamed mucosa and adjacent mucosa of such patients, which can assume that this expression pattern may differentiate CD from UC. By comparing UC tissue to adjacent normal tissue but alo to normal mucosa from healthy controls, all analyzed miRNAs in this study were significantly overexpressed in UC tissue, with some limitations in differentiating UC and CD [3]. In addition, Mohammadi et al. identified a panel of ten miRNAs differentially expressed in CD patients in endoscopic remission compared to healthy control subjects, miR-874-3p making the difference between the UC and CD cases. Further studies are necessary for developing the method of using these miRNAs in obtaining the differentiated diagnosis, but they are still a useful tool for monitoring the disease progress [32].

Ye et al. based on the fact that circular RNAs (circRNAs) participate in the pathogenesis of a variety of diseases and are considered ideal biomarkers of human disease, conducted a study in which was examined the expression of circRNA_103516 in inflammatory bowel diseases and its associations with clinical phenotypes and inflammatory cytokines. Circular RNAs (circRNAs) are covalently closed continuous single-stranded RNA molecules that have advantageous properties because their circular structure enables rolling circle RNA replication and producing multiple 
genomic copies once a single initiation event occurs. However, the characteristics of circRNAs are much less clear than those of microRNAs (miRNAs) and long noncoding RNAs (lncRNAs). Nonetheless, research on their functions has recently emerged, and increasing evidence demonstrates that circRNAs can modulate gene expression at the transcriptional or post-transcriptional level by sponging miRNAs or by interacting with other molecules. Furthermore, circRNAs are evolutionally conserved, and their expression is relatively stable in the cytoplasm; these features indicate that circRNAs may be ideal biomarkers in human disease. CircRNAs are ideal diagnostic and therapeutic candidates due to their stability and conservation. In the study were involved 90 Crohn's disease (CD) patients and 90 ulcerative colitis (UC) patients, 80 HCs (healthy controls), and 35 PCs (patient controls). CircRNA_103516 was upregulated in CD and UC patients compared with HCs and PCs. In addition, circRNA_103516 levels were increased in active CD and UC compared with remittent groups. The conclusion of the study was that CircRNA_103516 levels in Peripheral blood mononuclear cells can be considered an ideal candidate biomarker for diagnosing IBD. Dysregulation of circRNA_103516 may participate in the molecular mechanism of IBD through hsa-miR19b-1-5p sponging [33].

\subsubsection{Galectins}

Galectins are a family of galactoside-binding proteins commonly altered in the circulation of disease conditions such as cancer and inflammation. Serum levels of galectins such as galectins-2,-3, -4 and -8 are significantly higher in colon cancer patients than in healthy people and promote the dissemination of circulating haematogenous tumour cell in metastasis [34].

$\mathrm{Yu}$ et al. analyzed levels of galectins-1, -2, -3, -4, -7 and -8 in 208 samples from ambulant IBD patients (97 CD, 71 UC) and 40 from healthy people. Serum levels of galectins- 1 and -3 , but not galectins $-2,-4,-7$ and -8 , are significantly elevated in both UC and CD patients in comparison to healthy people. The difference between the values of these galactins were not statistically significant to be able to separate active and inactive $\mathrm{UC}$ and $\mathrm{CD}$, but they have the potential to be developed as biomarkers for general IBD determination [34].

\subsubsection{Biomarkers from gut microbiota}

The study of Zhou et al. aimed to identify non-invasive biomarkers for distinguishing UC from CD, based on the gut microbiom. UC was characterized by the presence of Actinobacteria, Proteobacteria, Bacilli, Bifidobacteriaceae, Bifidobacteriales, Coriobacteriales, Enterococcus, Enterococcaceae, Streptococcus, Steptococcaceae, Lactobacillales, Enterobacteriaceae, Enterobacteriales, and Pseudomonadales while healthy controls (HC) were predominantly enriched in Prevotella, Prevotellaceae, Bacteroidales, Roseburia, Lachnospiraceae, Ruminococcaceae and Clostridiales [35].

Fusobacterium nucleatum was reported to contribute to colorectal carcinoma by promoting a favourable microenvironment for carcinoma progression in UC; it has also been suggested as a biomarker for diagnosis. Also Gardnerella vaginalis was significantly more enriched in fecal samples from patients with UC, showing a potential use of the gut microbiome to aid in UC diagnosis being of significant clinical value in the management of IBD; study mentioned the limitation offered by the difference of gut microbiota in pediatric or elderly patients who cannot be included in the pattern [35].

As an increasing number of children are affected by inflammatory bowel disease (IBD) worldwide, the need for a diagnosis method less invasive than repeated endoscopic test emerged. Furthermore, it is challenging to distinguish Crohn's disease from ulcerative colitis endoscopically. 
Tatsuki et al. conducted a study that aimed to determine a putative biomarker for distinguishing CD from UC in pediatric patients by scanning serological cytokine profile. They showed that an (interleukin) IL-1 receptor antagonist, IL-6, and macrophage inflammatory protein- $1 \beta$ were associated with pediatric UC activity index (PUCAI) compared with CD where the granulocyte-macrophage colony-stimulating factor was incriminated. Also growth-regulated oncogene and TNF- $\alpha$ were higher in UC than in controls. This study observed clear differences in cytokine profiles between CD and UC, with IL-7 alone having the highest diagnostic accuracy [36].

\subsubsection{Autoantibodies}

Hamanaka et al. identified 3 novel serum antibodies through Alpha LISA technique. They are anti-PARG (poly ADP-ribose glycohydrolase), anti-TCEAL 1 (transcription elongation factor A protein-like 1) and anti-PRR13 (proline-rich 13), which have been found in a large amount in UC patients comparative to CD patients. From all 3 antibodies, TCEAL 1 has been associated for the first time with UC. PARG antibody is a biomarker used for the evaluation of mucosa impairment by inflammation at patients with UC. An augmented level of PRR13 antibody is UC patients is related to an antiinflammatory process from angiogenesis through suppression of TSP-1 (thrombospondin-1). In this study has been also studied other markers: first is pANCA, which is a common antibody found in IBD, in a percentage of $60-70 \%$ at patients with $\mathrm{UC}$ and it is the result of a cross-reaction between it and intestinal bacterial antigens and the other one is CUZD 1 antibody, a unique marker for IBD patients [37].

Ulcerative colitis alongside with Crohn's disease are chronic and relapsing gastrointestinal disorders with an unclear etiology. In order for a better diagnosis for these conditions, a large range of serological antibodies were studied. The most important and widely used antibodies are ASCA (anti-Saccharomyces cerevisiae antibody) and ANCA (anti-neutrophil cytoplasmic antibody). Nevertheless new biomarkers were developed and can be included in this category the following antibodies: Escherichia coli outer membrane porin $C$ antibody (anti-OmpC), exocrine pancreatic antibody (PAB), intestinal goblet cells antibody (GAB), anti-glycan antibody, bacterial flagellin CBir1 antibody (anti-CBir1), and Pseudomonas fluorescens-associated sequence 12 antibody (antil2). In prospective study whose purpose was to was to assess the clinical value of multiple antibodies, 130 Crohn's disease (CD) and 120 ulcerative colitis (UC) patients, compared with 80 healthy subjects, were investigated and the results showed that are serviceable biomarkers for IBD and they can lead to a better clinical decision. However, the serological positive antibodies were more inclined to be diagnosed as CD rather than UC. Positivity of ANCA was associated with disease activity of UC and showed a sensitivity of $46.7 \%$. GAB (IgG) positivity implied extensive location in UC. A combination of ASCA/anti-OmpC IgA/GP2 (IgG) showed the best specificity in distinguishing CD from UC [38].

\subsubsection{Volatile organic compounds (VOC)}

VOC are gaseous molecules produced during bacterial fermentation in the gut and they give information about the gut microbiota status of the host and the interaction between the two. These compounds showed diagnosis utility in other conditions such as cancer and diabetes and IBD patients are proposed to be candidates for analyzing the benefits brought by them in establishing the diagnosis. VOC are able biomarkers to distinguish between active UC from remission UC and to evaluate the inflammation status in this disease [39].

Hassani et al. aimed to assess the potential of urinary and fecal VOC as biomarkers in both pediatric UC and CD by comparing with the endoscopic and histopathologic outcome. There is a significant difference between IBD and controls for both fecal and 
urinary VOC profiles ( $\mathrm{p}$-value, area under the curve; $0.038,0.73$ vs. $0.028,0.78$ ), making the analysis of these compounds, potential noninvasive markers in detecting ulcerative colitis. Also, as the urine collecting method can be faster and more easily accepted by the patients, this type of establishing a preliminary diagnosis can accelerate the process. The distinction between the two methods is that analyses of fecal VOCs is believed to reflect the gut microbiota composition and local inflammatory processes, on the contrary, urinary VOCs associated with IBD are more likely to be a combination of compounds that diffused from the intestine into the bloodstream and VOCs that are associated with general inflammation [40]. Bosch et al. previously used field asymmetric ion mobility spectrometry (FAIMS) to analyze fecal VOC composition as a potential tool in differentiating two gastro-intestinal conditions relying on the accessibility of the process in pediatric patients, achieving similar diagnostic accuracies [41].

Determination of volatile organic compounds from exhaled breath is a non-invasive method for the diagnosis of IBD. The first objective of the most studies was to determine whether exhaled VOCs can be used to distinguish IBD from healthy controls and CD from UC. The volatile organic compounds from exhaled breath are a combination of biproducts of normal metabolic activity and there is an estimate of over $3000 \mathrm{VOCs}$ in human breath. Ammonia, sulphur dioxide $\left(\mathrm{SO}_{2}\right)$ and nitrogen dioxide $\left(\mathrm{NO}_{2}\right)$ were the substances described in one study and it has been showed that they contributed the most to the separation between diagnostic groups. Important changes were observed in ammonia by both UC and CD patients. Changes in $\mathrm{NO}_{2}$ were greater in $\mathrm{CD}$ over UC and controls, while UC is associated with increased sulphur dioxide. Ethylene oxide in UC and controls showed small differences between groups. In other studies were identified 6 VOCs (hydrogen cyanide, ammonia, dimethyl sulphide, hydrogen sulphide, butanal, and nonanal) which significantly showed differences in concentrations, between diagnostic groups. Other analysis used VOCs (1-octene, 1-decene, (E)-2-nonene) as relevant for predicting the presence of IBD, but did not identify any significant difference in VOCs between CD and UC [42].

\subsubsection{Protein-linked carbohydrates}

Protein-linked carbohydrates can be used as biomarkers for UC diagnosis since now it is a need for noninvasive test for diagnose of inflammatory bowel diseases as UC and Crohn's disease. In a study was analyzed the profile of total plasma N-glycomes of 2635 patients with inflammatory bowel diseases. The plasma samples were analyzed by mass spectrometry with a linkage-specific sialic acid derivatization technique and were compared the profiles of the patients with Crohn's disease or UC and the profiles of the control group. The results showed a bigger number for large-size glycans in the samples from the patients with UC and Crohn's disease compared with control samples. In addition, it was observed a low number of hybrid and high-mannose structures, lower fucosylation, lower galactosylation, and higher sialylation (a 2,3- and a2,6-linked). A difference between patients with $\mathrm{CD}$ and patients with UC can be done based on the higher bisection, lower galactosylation, and higher sialylation (a 2,3-linked). Also, in this study it is showed that glycosylation models were associated with disease location and progression [43].

\subsubsection{Serum Fetuin A}

Fetuin A ( $\alpha 2$-heremans-schmid glycoprotein (AHSG)) is a multifunctional plasma glycoprotein with a molecular weight of approximately $60 \mathrm{kDa}$ and is a member of the cystatin superfamily. Fetuin A has been studied as an inhibitor of ectopic calcium deposition, an important promoter of insulin resistance, and a negative acute phase reactant. Acting as a negative acute phase protein, fetuin A binds macrophage deactivating cations and the resulting fetuin-cation complex also transmits an anti- 
inflammatory signal. Fetuin $\mathrm{A}$ is synthesized by hepatocytes and its concentration in the serum falls by about 10 -fold after birth. In humans, fetuin $\mathrm{A}$ is a negative acute phase protein, as serum concentrations are significantly reduced after major surgical procedures, trauma, burns, and severe inflammation. In one study which involved a total of 90 patients and 41 healthy individuals was found that fetuin A levels in both the $\mathrm{UC}$ and CD groups were significantly lower than in $\mathrm{HC}$; however, there was no difference between the UC and CD groups, this means that fetuin, this means that fetuin A test can be used to differentiate UC and CD patients from HC but can't be used to distinguish UC patients from CD patients [44].

\subsubsection{Transferrin}

Transferrin is a glycoprotein capable of reversible iron binding, which serves as its transporter between iron donating enterocytes and macrophages and accepting erythroblasts. Systemic levels of transferrin differently respond to anemia depending on its cause. Its utility as a diagnostic marker in IBD-associated anemia is under the mark sign since transferrin concentration varies also in response to inflammation and malnutrition. Matusiewicz et al. wanted to evaluate transferrin levels in a cohort of IBD patients and relate it to indices of inflammation, malnutrition, anemia, and oxidative stress. They begin from the hypothesis that a lack of specificity caused by its relation to those indices IBD-accompanying conditions might be advantageous and transferrin may better reflect the overall disease severity. In this study were examined 234 individuals: 97 individuals as healthy controls and 137 IBD patients: 63 with Crohn's disease (36 with active and 27 with inactive disease) and 74 with ulcerative colitis (28 with active and 46 with inactive disease). It was observed a significant decrease in transferrin levels in patients with active $\mathrm{CD}$ and $\mathrm{UC}$, without differences between these two forms. Transferrin in IBD patients negatively correlated with inflammatory indices and the strongest correlations was with IL- 6 and hsCRP in CD and IL-6, WBC, and TNF- $\alpha$ in UC, although the latter association was found exclusively in patients with active disease. It was also examined transferrin association with indices of nutritional status and anemia and it was found out that transferrin positively correlate with all examined indices (albumin, cholesterol, hemoglobin, hematocrit, RBC, and iron) in UC patients but only with albumin and iron in CD patients. Additional it was examined the associations with antioxidants: serum paraoxonase- 1 and erythrocyte catalase, glutathione peroxidase-1, and superoxide dismutase-1. Transferrin positively correlated with all of these parameters in CD patients but in case of UC patients exclusively with paraoxonase-1 [45].

3.2.13. Other studies regarding potential biomarkers for diagnosis of UC

During last five years, there have been studied many potential parameters for diagnosis or treatment of UC, but some of them illustrated several markers that could be used to diagnose UC or to differentiate between UC and CD. All these studies are reviewed in Table 2 . 
Table 2. Recent studies regarding the identification of new potential biomarkers in UC.

\begin{tabular}{|c|c|c|c|}
\hline Subjects & Analysis technique & Main results & References \\
\hline $\begin{array}{l}21 \text { UC subjects } \\
22 \text { CD subjects } \\
21 \text { non-IBD controls }\end{array}$ & qRT-PCR & $\begin{array}{l}\text { Identification of } 2 \text { new genes in subjects with UC: } \\
\text { CCL11 and MMP10 } \\
\text { Identification of } 2 \text { new genes in patients with CD: } \\
\text { C4BPB and IL1RN } \\
\text { These } 4 \text { new genes are potential candidate biomarkers } \\
\text { to differentiate UC from CD, which could direct the } \\
\text { researchers for future therapeutic targets. }\end{array}$ & [46] \\
\hline 80 UC subjects & ELISA & $\begin{array}{l}\text { An increased level of fecal concentration of sST2 to the } \\
\text { UC patients with higher endoscopic, clinical and total } \\
\text { Mayo score. SST2 could be a future biomarker for the } \\
\text { evaluation of UC severity and could open promising } \\
\text { perspectives as a therapeutic aim. }\end{array}$ & [47] \\
\hline $\begin{array}{l}106 \text { UC subjects } \\
21 \text { control subjects }\end{array}$ & WGCNA & $\begin{array}{l}\text { Identification a new potential tissue biomarkers for } \\
\text { diagnosis or treatment of UC: DUOXA2, SAA1, SAA2, } \\
\text { TNIP3, CXCL1, SLC6A14 and CD55; } \\
\text { DUOXA2 and SLC6A14 could be new targets for gene } \\
\text { therapy in UC; } \\
\text { The pathways DUOXA2/DUOX2 and CXCL1/CXCR2 } \\
\text { could have an essential role in the evolution of UC } \\
\text { through the inflammatory responses. }\end{array}$ & [48] \\
\hline Female C57BL/6JJ mice & $\begin{array}{l}\text { Serum-associated } \\
\text { modulation of } \\
\text { fluorescent P450 } \\
\text { inhibition assay }\end{array}$ & $\begin{array}{l}\text { It has been found that CYP219 and CYP2E1 from the } \\
\text { serum of UC model mice could be used as potential } \\
\text { biomarkers for assessment of UC. These simple } \\
\text { fluorescent P450 inhibition tests present the capacity } \\
\text { to act like a new procedure to diagnose UC in mouse, } \\
\text { also with promising results at human subjects. }\end{array}$ & [49] \\
\hline $\begin{array}{l}19 \text { pediatric and } 87 \\
\text { adult UC subjects }\end{array}$ & Gene ontology & $\begin{array}{l}\text { Identification of new potential biomarkers for UC } \\
\text { diagnosis and treatment and also their fundamental } \\
\text { roles in the pathological differences between UC at } \\
\text { children and adults: CDC42, POLR2A, RAC1, PIK3R1, } \\
\text { MAPK1 and SRC. }\end{array}$ & [50] \\
\hline $\begin{array}{l}48 \text { UC subjects } \\
96 \text { healthy controls }\end{array}$ & ELISA & $\begin{array}{l}\text { There have been analyzed } 2 \text { parameters to } \\
\text { differentiate the patients with UC from healthy } \\
\text { subjects: NLR and PLR. They also can be used as } \\
\text { biomarkers to diagnose UC and to evaluate the status } \\
\text { of gut mucosa, especially in patients where } \\
\text { colonoscopy is not possible. }\end{array}$ & [51] \\
\hline $\begin{array}{c}42 \text { IBD subjects } \\
18 \text { healthy subjects }\end{array}$ & ELISA & $\begin{array}{c}\text { There have been discovered two biomarkers for UC } \\
\text { diagnosis: LTA4H and CAT, identified from MLI } \\
\text { (mucosal-luminal interface) proteome. }\end{array}$ & [52] \\
\hline $\begin{array}{c}140 \text { UC subjects } \\
128 \text { CD subjects } \\
224 \text { subjects with } \\
\text { intestinal associated } \\
\text { diseases as controls }\end{array}$ & $\begin{array}{l}\text { ELISA } \\
\text { Immunofluorescent } \\
\text { assay }\end{array}$ & $\begin{array}{c}\text { It has been evaluated four biomarkers to diagnose UC } \\
\text { and CD: pANCA, PAB, ASCA and GAB. From all } \\
\text { these markers, pANCA and PAB showed a higher } \\
\text { level in UC and CD, while ASCA and GAB presented } \\
\text { insignificant levels in IBD. }\end{array}$ & [53] \\
\hline
\end{tabular}




\begin{tabular}{|c|c|c|c|}
\hline $\begin{array}{l}33 \text { UC subjects } \\
27 \text { CD subjects } \\
20 \text { healthy controls }\end{array}$ & ELISA & $\begin{array}{l}\text { Four biomarkers have been assessed as a potential } \\
\text { indicators used for the diagnosis and evaluation of UC } \\
\text { and CD clinical activity: NEU, HLE- } \alpha 1 \mathrm{AT} \text {, lactoferrin } \\
\text { and calprotectin. It have been resulted higher ratios of } \\
\text { HLE- } \alpha 1 \mathrm{AT} / \mathrm{NEU} \text { and lactoferrin/NEU in UC subjects } \\
\text { than with CD and higher levels of plasma calprotectin } \\
\text { and lactoferrin in CD subjects than with UC. }\end{array}$ & {$[54]$} \\
\hline 158 UC subjects & - & $\begin{array}{l}\text { It has been studied the NeuPla ratio as a potential } \\
\text { biomarker for diagnosis of UC, with better results than } \\
\text { serum biomarkers (CRP, ESR and albumin). }\end{array}$ & [55] \\
\hline
\end{tabular}

$\begin{array}{ll}\text { Abbreviations: } & \\ \text { ASCA } & \text { antibodies to saccharomyces cerevisiae } \\ \text { C4BPB } & \text { complement component } 4 \text { binding protein beta } \\ \text { CAT } & \text { catalase } \\ \text { CCL11 } & \text { C-C motif chemokine ligand 11 } \\ \text { CD55 } & \text { complement decay-accelerating factor (CD antigen CD55) } \\ \text { CDC42 } & \text { cell division control protein } 42 \\ \text { CRP } & \text { C-reactive protein } \\ \text { CXCL1 } & \text { C-X-C motif chemokine } \\ \text { CXCR2 } & \text { C-X-C chemokine receptor type 2 } \\ \text { DUOXA2 } & \text { dual oxidase maturation factor 2 } \\ \text { ESR } & \text { erythrocyte sedimentation rate } \\ \text { GAB } & \text { antibodies against intestinal goblet cells } \\ \text { HLE- } \boldsymbol{\alpha} \text { IAT } & \text { leukocytes elastase-alfa 1 antytripsine complex } \\ \text { IL1RN } & \text { interleukin 1 receptor antagonist } \\ \text { LTA4H } & \text { leukotriene A-4 hydrolase } \\ \text { MAPK1 } & \text { mitogen-activated proyein konase 1 } \\ \text { MMP10 } & \text { matrix metallopeptidase 10 } \\ \text { NEU } & \text { neutrophils } \\ \text { NeuPla ratio } & \text { Neutrophil-Platelet ratio } \\ \text { NLR } & \text { neutrophil to lymphocyte ratio } \\ \text { PAB } & \text { pancreatic antibodies } \\ \text { pANCA } & \text { antibodies to perinuclear anti-neutrophil cytoplasmic } \\ \text { PIK3R1 } & \text { phosphatidylinositol 3-kinase regulatory subunit alpha } \\ \text { PLR - } & \text { platelet to lymphocyte ratio } \\ \text { POLR2A } & \text { NDA-directed RNA polymerase II subunit RPB1 } \\ \text { qRT-PCR } & \text { quantitative reverse transcription polymerase chain reaction } \\ \text { RAC1 } & \text { Ras-related C3 botulinum toxin substrate 1 } \\ \text { SAA1 and SAA2 } & \text { serum amyloid A 1 and 2 } \\ \text { SLC6A14 } & \text { solute carrier family 6 member 14 } \\ \text { SRC } & \text { proto-oncogene tyrosine-protein kinase Src } \\ \text { sST2 } & \text { soluble protein ST2, ELISA - enzyme-linked immunosorbent assay } \\ \text { TNIP3 - } & \text { TNFAIP3-interacting protein 3 } \\ \text { WGCNA } & \text { weighted gene coexpression network analysis } \\ & \end{array}$

\section{Conclusions}

Ulcerative colitis is an inflammatory bowel disease that causes inflammation and ulcers in the digestive tract, and sometimes can lead to complications. Since treatment can reduce signs and symptoms of the disease, it is very important to make an accurate diagnosis at an early stage. Despite the highly prevalence rate, the pathogenesis of UC is not fully elucidated and as a result, treatment does not include a wide range of medicines, the need for precision in choosing the suitable medicine being mandatory. Also the most used and accurate pathway for diagnosis is endoscopy, which is an invasive, time-consuming, expensive and painful method [56]. 
Biomarkers from stool and serum are non-invasive markers used as new targets of diagnosis, which have the advantages to be reproducible, accurate, standardized and to have sensitivity and specificity. The capacity to determine the type of $\mathrm{UC}$, to make difference between UC and Crohn's disease, to determinate the severity and the response to therapy using markers are the major purpose of studies regarding biomarkers used in diagnosis of UC.

Studies that were analyzed in this review showed suitable non-invasive biomarkers for diagnosis and some of them indicate also highly sensitivity and specificity. However, for a better characterization of these biomarkers it is mandatory to increase the number of studies in order to demonstrate their real utility [57].

Funding: This research received no external funding

Institutional Review Board Statement: Not applicable.

Informed Consent Statement: Not applicable.

Conflicts of Interest: The authors declare no conflict of interest.

\section{References}

1. Zhou, Y.; Xu, Z.Z.; He, Y.; Yang, Y.; Liu, L.; Lin, Q.; Nie, Y.; Li, M.; Zhi, F.; Liu, S., et al. Gut Microbiota Offers Universal Biomarkers across Ethnicity in Inflammatory Bowel Disease Diagnosis and Infliximab Response Prediction. mSystems 2018, 3, doi:10.1128/mSystems.00188-17.

2. Arafah, K.; Kriegsmann, M.; Renner, M.; Lasitschka, F.; Fresnais, M.; Kriegsmann, K.; von Winterfeld, M.; Goeppert, B.; Kriegsmann, J.; Casadonte, R., et al. Microproteomics and Immunohistochemistry Reveal Differences in Aldo-Keto Reductase Family 1 Member C3 in Tissue Specimens of Ulcerative Colitis and Crohn's Disease. Proteomics Clin. Appl. 2020, 14, e1900110, doi:10.1002/prca.201900110.

3. Guz, M.; Dworzanski, T.; Jeleniewicz, W.; Cybulski, M.; Kozicka, J.; Stepulak, A.; Celinski, K. Elevated miRNA Inversely Correlates with E-cadherin Gene Expression in Tissue Biopsies from Crohn Disease Patients in contrast to Ulcerative Colitis Patients. Biomed Res Int 2020, 2020, 4250329, doi:10.1155/2020/4250329.

4. Fernández-Tomé, S.; Montalban-Arques, A.; Díaz-Guerra, A.; Galvan-Roman, J.M.; Marin, A.C.; Mora-Gutiérrez, I.; Ortega Moreno, L.; Santander, C.; Sánchez, B.; Chaparro, M., et al. Peptides encrypted in the human intestinal microbial-exoproteome as novel biomarkers and immunomodulatory compounds in the gastrointestinal tract. J. Funct. Foods 2019, 52, 459-468, doi:10.1016/j.jff.2018.11.036.

5. Motooka, K.; Morishita, K.; Ito, N.; Shinzaki, S.; Tashiro, T.; Nojima, S.; Shimizu, K.; Date, M.; Sakata, N.; Yamada, M., et al. Detection of fucosylated haptoglobin using the 10-7G antibody as a biomarker for evaluating endoscopic remission in ulcerative colitis. World J. Gastroenterol. 2021, 27, 162-175, doi:10.3748/wjg.v27.i2.162.

6. Matsubayashi, M.; Kobayashi, T.; Okabayashi, S.; Nakano, M.; Sagami, S.; Ozaki, R.; Kiyohara, H.; Morikubo, H.; Asonuma, K.; Miyatani, Y., et al. Determining the usefulness of Capsule Scoring of Ulcerative Colitis in predicting relapse of inactive ulcerative colitis. J. Gastroenterol. Hepatol. 2021, 36, 943-950, doi:10.1111/jgh.15220.

7. Fabian, O.; Hradsky, O.; Lerchova, T.; Mikus, F.; Zamecnik, J.; Bronsky, J. Limited clinical significance of tissue calprotectin levels in bowel mucosa for the prediction of complicated course of the disease in children with ulcerative colitis. Pathol. Res. Pract. 2019, 215, 152689, doi:10.1016/j.prp.2019.152689.

8. Tsuda, S.; Carreras, J.; Kikuti, Y.Y.; Nakae, H.; Dekiden-Monma, M.; Imai, J.; Tsuruya, K.; Nakamura, J.; Tsukune, Y.; Uchida, T., et al. Prediction of steroid demand in the treatment of patients with ulcerative colitis by immunohistochemical analysis of the mucosal microenvironment and immune checkpoint: role of macrophages and regulatory markers in disease severity. Pathol. Int. 2019, 69, 260-271, doi:10.1111/pin.12794.

9. Xia, D.; An, J. Serum folic acid, PCT, CRP and ESR detection as biomarker in differentiation ulcerative colitis activity. Pteridines 2018, 29, 179-186, doi:10.1515/pteridines-2018-0016.

10. Yamamoto, M.; Shanmuganathan, M.; Hart, L.; Pai, N.; Britz-McKibbin, P. Urinary Metabolites Enable Differential Diagnosis and Therapeutic Monitoring of Pediatric Inflammatory Bowel Disease. Metabolites 2021, 11, doi:10.3390/metabo11040245.

11. Zhou, R.; Qiu, P.S.; Wang, H.Z.; Yang, H.J.; Yang, X.Y.; Ye, M.L.; Wang, F.; Zhao, Q. Identification of microRNA-16-5p and microRNA-21-5p in feces as potential noninvasive biomarkers for inflammatory bowel disease. Aging-US 2021, 13, 4634-4646.

12. Angelidou, I.; Chrysanthopoulou, A.; Mitsios, A.; Arelaki, S.; Arampatzioglou, A.; Kambas, K.; Ritis, D.; Tsironidou, V.; Moschos, I.; Dalla, V., et al. REDD1/Autophagy Pathway Is Associated with Neutrophil-Driven IL-1beta Inflammatory Response in Active Ulcerative Colitis. J. Immunol. 2018, 200, 3950-3961, doi:10.4049/jimmunol.1701643.

13. Hong, Z.-C.; Cai, Q.; Wu, H.-Z.; Yang, Y.-F.; Fan, H.; Duan, X.-Y. Compound Sophorae Decoction: treating ulcerative colitis by affecting multiple metabolic pathways. Chinese Journal of Natural Medicines 2021, 19, 267-283, doi:10.1016/s1875-5364(21)600298 . 
14. Tang, Q.; Cang, S.; Jiao, J.; Rong, W.; Xu, H.; Bi, K.; Li, Q.; Liu, R. Integrated study of metabolomics and gut metabolic activity from ulcerative colitis to colorectal cancer: The combined action of disordered gut microbiota and linoleic acid metabolic pathway might fuel cancer. J. Chromatogr. A 2020, 1629, 461503, doi:10.1016/j.chroma.2020.461503.

15. Li, X.; Lee, E.J.; Gawel, D.R.; Lilja, S.; Schafer, S.; Zhang, H.; Benson, M. Meta-Analysis of Expression Profiling Data Indicates Need for Combinatorial Biomarkers in Pediatric Ulcerative Colitis. J Immunol Res 2020, 2020, 8279619, doi:10.1155/2020/8279619.

16. Olbjorn, C.; Cvancarova Smastuen, M.; Thiis-Evensen, E.; Nakstad, B.; Vatn, M.H.; Perminow, G. Serological markers in diagnosis of pediatric inflammatory bowel disease and as predictors for early tumor necrosis factor blocker therapy. Scand. J. Gastroenterol. 2017, 52, 414-419, doi:10.1080/00365521.2016.1259653.

17. Daniluk, U.; Daniluk, J.; Kucharski, R.; Kowalczyk, T.; Pietrowska, K.; Samczuk, P.; Filimoniuk, A.; Kretowski, A.; Lebensztejn, D.; Ciborowski, M. Untargeted Metabolomics and Inflammatory Markers Profiling in Children With Crohn's Disease and Ulcerative Colitis-A Preliminary Study. Inflamm. Bowel Dis. 2019, 25, 1120-1128, doi:10.1093/ibd/izy402.

18. Honig, G.; Heller, C.; Hurtado-Lorenzo, A. Defining the Path Forward for Biomarkers to Address Unmet Needs in Inflammatory Bowel Diseases. Inflamm. Bowel Dis. 2020, 26, 1451-1462, doi:10.1093/ibd/izaa210.

19. Moniuszko, A.; Głuszek, S.; Rydzewska, G.J.P.A.I.M. Rapid fecal calprotectin test for prediction of mucosal inflammation in ulcerative colitis and Crohn disease: a prospective cohort study. 2017, 127, 312-318.

20. Tefas, C.; Ciobanu, L.; Tanțău, M.; Moraru, C.; Socaciu, C.J.B.j.o.b.m.s. The potential of metabolic and lipid profiling in inflammatory bowel diseases: A pilot study. 2020, 20, 262.

21. De Arce, E.P.; Sedano, R.; Quera, R. Biomarkers in inflammatory bowel disease. Rev. Med. Chil. 2020, 148, 362-370.

22. Mihai, C.; Cijevschi Prelipcean, C.; Dranga, M.; Gavrilescu, O.; Cardoneanu, A.; Lacatusu, C.; Mihai, B.M. Correlations Between Inflammatory Biomarkers and Activity in Inflammatory Bowel Diseases. Rev. Chim. 2018, 69, 710-713, doi:10.37358/rc.18.3.6181.

23. Gur, E.S.; Cindemir, O. THE COMBINED PERFORMANCE OF FECAL CALPROTECTIN WITH FECAL OCCULT BLOOD, LYMPHOCYTE AND NEUTROPHIL PERCENTAGES IN DISCRIMINATING ULCERATIVE COLITIS. Nobel Med. 2020, 16, 13-21.

24. Galipeau, H.J.; Caminero, A.; Turpin, W.; Bermudez-Brito, M.; Santiago, A.; Libertucci, J.; Constante, M.; Raygoza Garay, J.A.; Rueda, G.; Armstrong, S., et al. Novel Fecal Biomarkers That Precede Clinical Diagnosis of Ulcerative Colitis. Gastroenterology 2021, 160, 1532-1545, doi:10.1053/j.gastro.2020.12.004.

25. Karling, P.; Lundgren, D.; Eklof, V.; Palmqvist, R.; Hultdin, J. Improved monitoring of inflammatory activity in patients with ulcerative colitis by combination of faecal tests for haemoglobin and calprotectin. Scand. J. Clin. Lab. Invest. 2019, 79, 341-346, doi:10.1080/00365513.2019.1622148.

26. Jusue, V.; Chaparro, M.; Gisbert, J.P. Accuracy of fecal calprotectin for the prediction of endoscopic activity in patients with inflammatory bowel disease. Dig. Liver Dis. 2018, 50, 353-359, doi:10.1016/j.dld.2017.12.022.

27. Whitehead, S.J.; Ford, C.; Gama, R.M.; Ali, A.; McKaig, B.; Waldron, J.L.; Steed, H.; Brookes, M.J. Effect of faecal calprotectin assay variability on the management of inflammatory bowel disease and potential role of faecal S100A12. J. Clin. Pathol. 2017, 70, 1049-1056, doi:10.1136/jclinpath-2017-204340.

28. Mak, L.Y.; Tong, T.S.M.; Cheung, K.S.; Chen, L.J.; Lui, K.L.; Lau, K.S.; Leung, W.K. Combined Use of Common Fecal and Blood Markers for Detection of Endoscopically Active Inflammatory Bowel Disease. Clin. Transl. Gastroenterol. 2020, 11, 8, doi:10.14309/ctg.0000000000000138.

29. Miao, Z.; Ding, K.; Jin, S.; Dai, L.; Dai, C.; Li, X. Using serum peptidomics to discovery the diagnostic marker for different stage of ulcerative colitis. J. Pharm. Biomed. Anal. 2021, 193, 113725, doi:10.1016/j.jpba.2020.113725.

30. Sayar, S.; Kurbuz, K.; Kahraman, R.; Caliskan, Z.; Atalay, R.; Ozturk, O.; Doganay, H.L.; Ozdil, K. A practical marker to determining acute severe ulcerative colitis: CRP/albumin ratio. North Clin Istanb 2020, 7, 49-55, doi:10.14744/nci.2018.78800.

31. Viennois, E.; Zhao, Y.; Han, M.K.; Xiao, B.; Zhang, M.; Prasad, M.; Wang, L.; Merlin, D. Serum miRNA signature diagnoses and discriminates murine colitis subtypes and predicts ulcerative colitis in humans. Sci. Rep. 2017, 7, 2520, doi:10.1038/s41598017-02782-1.

32. Mohammadi, A.; Kelly, O.B.; Filice, M.; Kabakchiev, B.; Smith, M.I.; Silverberg, M.S. Differential Expression of microRNAs in Peripheral Blood Mononuclear Cells Identifies Autophagy and TGF-Beta-Related Signatures Aberrantly Expressed in Inflammatory Bowel Disease. Journal of Crohns \& Colitis 2018, 12, 568-581, doi:10.1093/ecco-jcc/jjy010.

33. Ye, Y.L.; Yin, J.; Hu, T.; Zhang, L.P.; Wu, L.Y.; Pang, Z. Increased circulating circular RNA_103516 is a novel biomarker for inflammatory bowel disease in adult patients. World J. Gastroenterol. 2019, 25, 6273-6288, doi:10.3748/wjg.v25.i41.6273.

34. Yu, T.B.; Dodd, S.; Yu, L.G.; Subramanian, S. Serum galectins as potential biomarkers of inflammatory bowel diseases. PLoS One 2020, 15, 12, doi:10.1371/journal.pone.0227306.

35. Zhou, Y.L.; He, Y.; Liu, L.; Zhou, W.Y.; Wang, P.; Hu, H.; Nie, Y.Q.; Chen, Y. Alterations in Gut Microbial Communities Across Anatomical Locations in Inflammatory Bowel Diseases. Front. Nutr. 2021, 8, 10, doi:10.3389/fnut.2021.615064.

36. Tatsuki, M.; Hatori, R.; Nakazawa, T.; Ishige, T.; Hara, T.; Kagimoto, S.; Tomomasa, T.; Arakawa, H.; Takizawa, T. Serological cytokine signature in paediatric patients with inflammatory bowel disease impacts diagnosis. Sci. Rep. 2020, 10, 11, doi:10.1038/s41598-020-71503-y. 
37. Hamanaka, S.; Nakagawa, T.; Hiwasa, T.; Ohta, Y.; Kasamatsu, S.; Ishigami, H.; Taida, T.; Okimoto, K.; Saito, K.; Maruoka, D., et al. Investigation of novel biomarkers for predicting the clinical course in patients with ulcerative colitis. J. Gastroenterol. Hepatol. 2018, 33, 1975-1983, doi:10.1111/jgh.14297.

38. Ye, Y.L.; Zhang, L.P.; Hu, T.; Chen, W.C.; Pang, Z. Prospective value of serologic antibodies in Chinese patients with inflammation bowel disease. Int. J. Clin. Exp. Med. 2019, 12, 4860-4869.

39. Smolinska, A.; Bodelier, A.G.; Dallinga, J.W.; Masclee, A.A.; Jonkers, D.M.; van Schooten, F.J.; Pierik, M.J. The potential of volatile organic compounds for the detection of active disease in patients with ulcerative colitis. Aliment. Pharmacol. Ther. 2017, 45, 1244-1254, doi:10.1111/apt.14004.

40. el Hassani, S.E.; Bosch, S.; Lemmen, J.P.M.; Brentar, M.B.; Ayada, I.; Wicaksono, A.N.; Covington, J.A.; Benninga, M.A.; de Boer, N.K.H.; de Meij, T.G.J. Simultaneous Assessment of Urinary and Fecal Volatile Organic Compound Analysis in De Novo Pediatric IBD. Sensors 2019, 19, 11, doi:10.3390/s19204496.

41. Bosch, S.; van Gaal, N.; Zuurbier, R.P.; Covington, J.A.; Wicaksono, A.N.; Biezeveld, M.H.; Benninga, M.A.; Mulder, C.J.; de Boer, N.K.H.; de Meij, T.G.J. Differentiation Between Pediatric Irritable Bowel Syndrome and Inflammatory Bowel Disease Based on Fecal Scent: Proof of Principle Study. Inflamm. Bowel Dis. 2018, 24, 2468-2475, doi:10.1093/ibd/izy151.

42. Tiele, A.; Wicaksono, A.; Kansara, J.; Arasaradnam, R.P.; Covington, J.A. Breath Analysis Using eNose and Ion Mobility Technology to Diagnose Inflammatory Bowel Disease-A Pilot Study. Biosensors-Basel 2019, 9, 15, doi:10.3390/bios9020055.

43. Clerc, F.; Novokmet, M.; Dotz, V.; Reiding, K.R.; de Haan, N.; Kammeijer, G.S.M.; Dalebout, H.; Bladergroen, M.R.; Vukovic, F.; Rapp, E., et al. Plasma N-Glycan Signatures Are Associated With Features of Inflammatory Bowel Diseases. Gastroenterology 2018, 155, 829-843, doi:10.1053/j.gastro.2018.05.030.

44. Karakoyun, I.; Isman, F.K.; Basok, B.I.; Arslan, F.D.; Calik, B.; Kahraman, N.G.; Akbulut, G.; Ulasoglu, C. Serum Fetuin A and Secreted Phosphoprotein 24 as Diagnostic Markers in Inflammatory Bowel Disease. J. Basic Clin. Health Sci. 2020, 4, 318-323, doi:10.30621/jbachs.2020.1123.

45. Matusiewicz, M.; Neubauer, K.; Lewandowska, P.; Gamian, A.; Krzystek-Korpacka, M. Reduced Transferrin Levels in Active Inflammatory Bowel Disease. Biomed Research International 2017, 2017, 8, doi:10.1155/2017/9541370.

46. Dobre, M.; Milanesi, E.; Manuc, T.E.; Arsene, D.E.; Tieranu, C.G.; Maj, C.; Becheanu, G.; Manuc, M. Differential Intestinal Mucosa Transcriptomic Biomarkers for Crohn's Disease and Ulcerative Colitis. J Immunol Res 2018, 2018, 9208274, doi:10.1155/2018/9208274.

47. Jovanovic, M.; Gajovic, N.; Jurisevic, M.; Simovic-Markovic, B.; Maric, V.; Jovanovic, M.; Arsenijevic, N.; Zdravkovic, N. Fecal sST2 correlates with the disease severity of ulcerative colitis. Vojnosanit. Pregl. 2019, 76, 1153-1161, doi:10.2298/vsp171225026j.

48. Zhang, J.; Wang, X.; Xu, L.; Zhang, Z.; Wang, F.; Tang, X. Investigation of Potential Genetic Biomarkers and Molecular Mechanism of Ulcerative Colitis Utilizing Bioinformatics Analysis. Biomed Res Int 2020, 2020, 4921387, doi:10.1155/2020/4921387.

49. Yamamoto, R.; Muroi, K.; Imaishi, H. Serum derived from ulcerative colitis mouse changes the metabolism of the fluorescent substrate by $\mathrm{P} 450$ depending on the degree of disease progression. Chem. Biol. Interact. 2018, 290, 88-98, doi:10.1016/j.cbi.2018.05.012.

50. Xiu, M.X.; Liu, Y.M.; Chen, G.Y.; Hu, C.; Kuang, B.H. Identifying Hub Genes, Key Pathways and Immune Cell Infiltration Characteristics in Pediatric and Adult Ulcerative Colitis by Integrated Bioinformatic Analysis. Dig. Dis. Sci. 2020, 10.1007/s10620-020-06611-w, doi:10.1007/s10620-020-06611-w.

51. Jeong, Y.; Jeon, S.R.; Kim, H.G.; Moon, J.R.; Lee, T.H.; Jang, J.Y.; Cho, J.-H.; Park, J.S.; Park, H.; Lee, K.-h.J.I.r. The role of platelet to lymphocyte ratio and neutrophil to lymphocyte ratio in ulcerative colitis. 2021, 19, 62.

52. Deeke, S.A.; Starr, A.E.; Ning, Z.; Ahmadi, S.; Zhang, X.; Mayne, J.; Chiang, C.K.; Singleton, R.; Benchimol, E.I.; Mack, D.R., et al. Mucosal-luminal interface proteomics reveals biomarkers of pediatric inflammatory bowel disease-associated colitis. Am. J. Gastroenterol. 2018, 113, 713-724, doi:10.1038/s41395-018-0024-9.

53. Zhang, S.; Luo, J.; Li, J.; Wu, Z.; Hu, C.; Li, P.; Deng, C.; Zhang, F.; Qian, J.; Li, Y. Retrospective evaluation of the clinical utility of serological biomarkers in Chinese patients with inflammatory bowel disease: 2-year clinical experience. Clin. Chem. Lab. Med. 2017, 55, 865-875, doi:10.1515/cclm-2016-0658.

54. Pawlica-Gosiewska, D.; Solnica, B.; Gawlik, K.; Cibor, D.; Mach, T.; Fedak, D.; Owczarek, D. The use of selected neutrophil protein plasma concentrations in the diagnosis of Crohn's disease and ulcerative colitis - a preliminary report. Postepy Hig Med Dosw (Online) 2017, 71, 243-253, doi:10.5604/01.3001.0010.3810.

55. Yamamoto-Furusho, J.K.; Mendieta-Escalante, E.A. Diagnostic utility of the neutrophil-platelet ratio as a novel marker of activity in patients with Ulcerative Colitis. PLoS One 2020, 15, e0231988, doi:10.1371/journal.pone.0231988.

56. Pereira, M.S.; Maia, L.; Azevedo, L.F.; Campos, S.; Carvalho, S.; Dias, A.M.; Albergaria, A.; Lima, J.; Marcos-Pinto, R.; Lago, P., et al. A [Glyco]biomarker that Predicts Failure to Standard Therapy in Ulcerative Colitis Patients. J. Crohns Colitis 2019, 13, 3949, doi:10.1093/ecco-jcc/jjy139.

57. Torres, J.; Petralia, F.; Sato, T.; Wang, P.; Telesco, S.E.; Choung, R.S.; Strauss, R.; Li, X.J.; Laird, R.M.; Gutierrez, R.L., et al. Serum Biomarkers Identify Patients Who Will Develop Inflammatory Bowel Diseases Up to 5 Years Before Diagnosis. Gastroenterology 2020, 159, 96-104, doi:10.1053/j.gastro.2020.03.007. 